Gdańsk 2019, Nr. 41

https://doi.org/10.26881/sgg.2019.41.16

Aleksander Baliński

PAN Biblioteka Gdańska / Bibliothek der Polnischen Akademie der Wissenschaften in Gdańsk

https://orcid.org/0000-0001-5774-6637

\title{
Die verlorene Seele einer Stadt. Danzig zwischen der Freien Stadt und den Revolutionen 1848/1849
}

Über Jahrhunderte hatte die an der Weichselmündung lebende Danziger Bevölkerung ein charakteristisches, souveränes Gemeinwesen hervorgebracht. Nach der erneuten Besetzung Danzigs durch die Preußen 1814 bewirkte die dezidiert antipreußische Gesinnung der Stadtbewohner gewisse Zugeständnisse seitens der Berliner Regierung. Obwohl die lokalen Zivilrechte beinahe ein halbes Jahrhundert ihre Gültigkeit behalten durften, schritt der wirtschaftliche Niedergang der Stadt, die während der Napoleonischen Kriege sehr verarmt war und bis 1857 Schulden zurückzahlen musste, voran. Noch in den 1830er Jahren identifizierte sich die Mehrheit der Danziger nicht mit Preußen. In den nachfolgenden Generationen machte sich aber ein Integrationsprozess bemerkbar, für den die Schulpflicht, der Wehrdienst und die Zuwanderung aus umliegenden Gebieten verantwortlich waren. Um die Mitte des 19. Jahrhunderts war die lokale Identität vermutlich in zwei Hälften gespalten: Einerseits gab es die Alt-Danziger, andererseits - die zugewanderten Anhänger der „preußischen Option“. Die Zukunft gehörte den Letzteren, auch wenn die Ersteren noch zu Beginn des 20. Jahrhunderts an der Mottlau anzutreffen waren.

Schlüsselwörter: Danzig, Geschichte des 19. Jahrhunderts; Danzig, Gesellschaft des 19. Jahrhunderts; erste (napoleonische) Freie Stadt Danzig, Revolutionen 1848/1849, lokale Identität

The lost soul of a city. Danzig between the Free City and the Revolutions of 1848. - Over the centuries people living in Danzig established a characteristic, sovereign urban republic located at the mouth of the Mottlau River. After the re-incorporation of the city-state into the Kingdom of Prussia in 1814, the strong anti-Prussian attitude of inhabitants of Danzig caused some concessions on the part of Berlin authorities. Although the local civil law remained valid for almost half of a century, the city suffered an economic decline caused by the Napoleonic wars and by the debts which were to be paid off until 1857. In the 1830s, the majority of citizens of Danzig did not identify themselves with Prussia. However, the integration of next generations was a continuous process because of compulsory school education, military service and immigration from outside. In the middle of the $19^{\text {th }}$ century, there probably existed two kinds of local identity, one of which constituted "old" Danzig citizens and the other: immigrants opting for Prussia. The

1 Die polnische Erstfassung dieses Beitrags unter dem Titel „Utracona dusza miasta. Gdańsk między Wolnym Miastem a Wiosną Ludów“ erschien im Katalog der Ausstellung Gdańsk i okolice 1793-1914. Miasto - ludzie wydarzenia wrysunku igrafice [Danzig und Umgebung 1793-1914. Stadt - Menschen - Ereignisse in Zeichnungen und Grafiken], die vom 26. Juli bis zum 14. September 2014 im Nationalmuseum in Gdańsk stattfand (vgl. BoNISŁAWSKI / BALIŃSKI (Hg.) 2014: 21-26). 
future belonged to the latter, although the former one could also still be found at the Mottlau River at the beginning of the $20^{\text {th }}$ century.

Keywords: Danzig, history of the $19^{\text {th }}$ century; Danzig, the society of the $19^{\text {th }}$ century; the Free City of Danzig (Napoleonic), local identity

Über Jahrhunderte hatte die an der Weichselmündung lebende Danziger Bevölkerung ein charakteristisches, souveränes Gemeinwesen hervorgebracht. Jegliche Identifikation mit den Preußen lehnten die Danziger dezidiert ab. Nach der ersten Teilung Polen-Litauens 1772 und der darauffolgenden Wirtschaftssperre, die Preußen über die Stadt verhängte, beschrieb Johanna Schopenhauer (1766-1838) die damalige Stimmung in Danzig folgendermaßen:

„Der Zorn der Bürger, den das Gefühl ihrer Ohnmacht bis zu verzweiflungsvoller Wuth erhöht hatte, wandelte, als der erste Schrecken überstanden war, in verbissenen Ingrimm, in immer tiefer eingreifenden $\mathrm{Ha} ß$ gegen Preußen und Alles, was Preußisch war, sich um, der bald in den festen Entschluß überging, zur Vertheidigung des letzten armseligen Scheines ehemaliger Freiheit, der ihnen geblieben war, alles daran zu setzen, Leib und Leben, Hab und Gut." (SCHOpenhauer 1884: 44)

Der Kampf um die Zukunft der Stadt verlief im Zeichen der Verteidigung der republikanischen Freiheiten, die man durch die Einverleibung in das absolutistische Königreich Preußen gefährdet sah. Die Danziger verbanden sich in dieser gemeinsamen Sache, dem Widerstand lagen jedoch vielfältige Motive zugrunde.

Die herrschenden Oberschichten beharrten auf ihrem Privileg, lukrative Posten zu bekleiden, die nun von preußischen Beamten übernommen werden sollten. Handwerker und Kaufleute, in Zünften und Körperschaften organisiert, sahen ihre Interessen durch die preußische Konkurrenz im Handel und in der Herstellung von Waren gefährdet (vgl. BIERNAT 1993: 72-74). Auch die unteren Gesellschaftsschichten, die die Stadtväter in deren Selbstbestimmungspolitik bisher unterstützt hatten, fürchteten die Folgen der preußischen Verwaltung. Was sie besonders beunruhigte, war der obligatorische Militärdienst, der in den von Preußen geführten Kriegen Gefahr für Leib und Leben mit sich brachte.

Nur wenige Danziger waren bereit, die Herrschaft Berlins zu akzeptieren - vor allem die Ärmeren, die mit ihren alltäglichen Sorgen beschäftigt waren. Einige vermögende Danziger waren ebenfalls der Meinung, dass sich die Stadt nicht richtig entwickeln könne, wenn die preußische Wirtschaftssperre nicht abgeschafft wird und Danzig seinen natürlichen Kontakt mit dem Weichsel-Hinterland wieder aufbaut (vgl. BIERNAT 1993: 72).

\section{Erste Generation: 1793-1814}

Als König Friedrich Wilhelm II. am 24. Februar 1793 ankündigte, die Stadt Danzig und ihr Umland zu besetzen, war er nicht besonders darum bemüht, die wenigen Anhänger Berlins für sich zu gewinnen. Die Okkupation begründete er demnach auch nicht mit den bestehenden engen Verbindungen zwischen Danzig und den umliegenden Gebieten, die nun dem 
Königreich Preußen zufallen sollten. Stattdessen erklärte er, die Stadt aufgrund ihrer antipreuBischen Haltung und auch wegen einer politischen Gefährdung durch die revolutionären, antimonarchistischen französischen Jakobiner besetzen zu müssen. Diese Umstände

„haben die Aufmerksamkeit S.K.M. auf diese Stadt lenken müssen, und Ihnen die Verbindlichkeit aufgelegt, sie in billigen Schranken zu halten, und solchergestalt für die Sicherheit und Ruhe Ihrer benachbarten Länder zu sorgen." ${ }^{\text {" }}$

Die Besetzung Danzigs am 4. April 1793 löste eine allgemeine Trauer unter den Einwohnern aus. Die Stadt verlor ihre umfangreichen Privilegien, die sie zur Zeit der altpolnischen Adelsrepublik genossen hatte; die bisherige städtische Selbstbestimmung musste einer zentralistischen Verwaltung weichen. Die Entschlossenheit und die Bereitschaft der Danziger, ihre angestammten Rechte mit Waffengewalt zu verteidigen, bewirkten jedoch gewisse Zugeständnisse auf Seiten Berlins: Die Stadt durfte ihre wirtschaftliche Autonomie beibehalten, und ihre Bewohner wurden vom Militärdienst freigestellt (vgl. BIERNAT 1993: 74).

Trotz der negativen Einstellung der Danziger gegenüber der preußischen Herrschaft blieb das Komplott des jungen Gottfried Benjamin Bartholdy, das dieser gemeinsam mit einer Gruppe von Handwerkslehrlingen und Schülern des Akademischen Gymnasiums schmiedete, der einzige Versuch, gegen die neue Ordnung in der Stadt zu rebellieren. Da die Verschwörung nur geringen Umfang hatte und der geplante Aufstand unzureichend vorbereitet war, wurde er 1797 im Keim erstickt. Die preußischen Besatzer entschlossen sich, etwaige Nachfolger Bartholdys durch drakonische Strafen abzuschrecken, die den Revolutionären zunächst auferlegt, schließlich aber weitgehend gemildert wurden (vgl. GŁ̨̨вOw ICZ 1961).

Die Berliner Regierung war darum bemüht, die antipreußische Gesinnung der Danziger aufzulösen. Dazu sollte der Besuch des neuen Königs Friedrich Wilhelm III. beitragen. Ende Mai bis Anfang Juni 1798 weilte dieser zusammen mit seiner Gemahlin, der schönen Königin Luise, in Danzig. Aus diesem Anlass wurden mehrtägige Feierlichkeiten veranstaltet, an denen verdiente Danziger Bürger zusammen mit höheren preußischen Beamten teilnahmen und dabei mit hohen Würden beschenkt wurden. Dennoch konnte die Generation, die die preußische Wirtschaftssperre erlebt hatte, ihre Leiderfahrung und die ihr einst zugefügten Demütigungen nicht vergessen (vgl. BIERNAT 1993: 74-75). ${ }^{3}$

Ein Teil der damaligen Danziger Bevölkerung stand unter dem Einfluss der liberalen Ideen der Französischen Revolution. Wer sich zum Motto „Freiheit, Gleichheit und Brüderlichkeit" bekannte, begehrte gegen den preußischen Absolutismus auf. Infolgedessen mussten zahlreiche Rebellen nach Westeuropa auswandern, darunter in die norddeutschen Länder - ein Schicksal, das unter anderem der Familie des Philosophen Arthur Schopenhauer beschieden war.

\footnotetext{
2 Declaration Seiner Majestät des Königs von Preussen, die Besetzung der Stadt und des Gebiets von Danzig betreffend. Vgl. auch AskenAZy (1923: 88-89).

3 Die missliche Lage der Danziger während der preußischen Besatzung entlud sich in heftiger Kritik an der Grobheit und schlechten Kinderstube der preußischen Beamten und Offiziere.
} 


\section{Die erste (napoleonische) Freie Stadt Danzig}

Der französisch-preußische Krieg 1806-1807 endete mit einer unerwarteten Niederlage des Königreichs Preußen; am 27. Mai 1807 wurde Danzig von den Franzosen erobert. Der siegreiche Napoleon Bonaparte beschloss, die Hafenstadt an der Mottlau zu seinem Stützpunkt an der Ostsee zu machen. Ein Mittel dazu war die Errichtung einer Freien Stadt Danzig aufgrund des Tilsiter Friedensvertrags von 1807. Formal unabhängig, stand der neue Stadtstaat unter dem Schutz der Könige von Preußen und Sachsen. Praktisch wurde er aber von dem französischen Gouverneur General Jean Rapp, Befehlshaber der städtischen Garnison, mitregiert (vgl. ROZENKRANZ 1980: 23-25).

Mit der wiedergewonnenen Freiheit verschlechterte sich die wirtschaftliche Lage Danzigs zusehends. Der Seehandel, der dem Wohlstand der lokalen Bevölkerung zugrunde lag, kam wegen der von Frankreich verfügten Kontinentalsperre, die den Warenaustausch mit Großbritannien verhinderte, zum Erliegen. Zudem wurden die Einwohner durch die von den Franzosen eingeforderten Kriegsabgaben und Kontributionen ruiniert. Den größten Schaden erlitten dabei die vermögenden Kaufleute, die sich für die Souveränität der Stadt einsetzten (vgl. ZaJEwSKi 1993a: 152-154, 164-167).

Napoleons gescheiterter Russlandfeldzug 1812 bereitete der ersten Freien Stadt Danzig ein Ende. Trotz der hartnäckigen Verteidigung durch die französischen und polnischen Truppen musste sich Danzig nach einer zehnmonatigen Belagerung, die für die Stadtbevölkerung verheerende Folgen hatte, am 2. Januar 1814 der russischen Armee ergeben. Dennoch gaben die Einwohner - zumindest die Danziger Patrizier - die Hoffnung nicht auf, die Unabhängigkeit ihrer Stadt beibehalten zu dürfen (vgl. WiLDER 1954: 507-510).

Am 8. Januar und am 23. März 1813 erhielt der diplomatische Agent der Freien Stadt Danzig in Paris, Dr. Wilhelm Daniel Keidel aus Bremen, von den Stadtobrigkeiten besondere Anweisungen. Bei den künftigen Friedensverhandlungen hatte er sich nämlich dafür einzusetzen, Danzigs politische und wirtschaftliche Bindungen an Polen aufrechtzuerhalten. Sollte ein mächtiges, unabhängiges Polen nicht restituiert werden können, so sollte man mit größter Aufmerksamkeit all das verfolgen, was der Aufrechterhaltung und Stärkung der politischen Eigenständigkeit des Danziger Staates dienlich sein könnte. Gleichzeitig hatte Keidel um die Unterstützung und die Gunst derjenigen Großmacht zu werben, welcher der Großteil der polnischen Lande an der Weichsel zufallen sollte (vgl. ZAJEwsKI 1993b: 190).

Keidel trat energisch dafür ein, die französischen, russischen und englischen Machthaber und Diplomaten für Danzigs Interessen zu gewinnen; dazu reiste er sogar zum Friedenskongress nach Wien. Trotzdem sprach sich keine der europäischen Großmächte - aus jeweils unterschiedlichen Gründen - für das Weiterbestehen der Freien Stadt Danzig aus (vgl. ebd.).

\section{Zweite Generation: 1815-1848}

Äußerst behutsam wurde die preußische Herrschaft in Danzig wieder eingeführt. Durch Kompromisspolitik versuchte man, den alten Groll der Danziger zu beschwichtigen. So hieß es im Aufruf des preußischen Gouverneurs Eberhard von Massenbach an die Danziger Bürger am 3. Februar 1814: 
„Achtbare Bürger der Stadt, Einwohner derselben und ihrer Umgebungen... Ihr kehret zu einem Staate zurück, werdet wieder Theil einer Nation... Ihr werdet als Preußen und mit Preußen Euch wieder glücklich sehen." ${ }^{“ 4}$

1817 wurden in Danzig die lokalen Zivilrechte, die sich von denen in anderen preußischen Staatsgebieten unterschieden, offiziell bestätigt; anschließend behielten sie noch nahezu fünfzig Jahre lang ihre Gültigkeit. Die Berliner Regierung beschloss, die Stadt Danzig allmählich in das Verwaltungssystem des preußischen Staates einzubinden - eine für ganz Preußen singuläre Entscheidung, die Danzig seiner politischen und strategischen Bedeutung verdankte (vgl. WŁodarCZy 1998a: 148).

Danzig befand sich in einem beklagenswerten Zustand, als es wieder unter preußische Herrschaft geriet. Es war insbesondere durch die Belagerungen von 1807 und 1813 regelrecht verwüstet. Die hohen Schulden aus der napoleonischen Zeit waren noch bis 1857 zurückzuzahlen. Der fortschreitende wirtschaftliche Niedergang der einst so mächtigen Stadt wurde allerdings in erster Linie durch die politischen Veränderungen in Mitteleuropa nach dem Wiener Kongress 1815 verursacht. Die Bürger von Danzig waren sich dessen bewusst und betrachteten die preußische Regierung mit Abneigung, zumal die Stadt bald ihr Prestige einbüßte, nachdem West- und Ostpreußen 1824 zur Provinz Preußen mit der Hauptstadt Königsberg vereinigt worden waren.

Ebenso wenig war zu erwarten, dass die preußische Wirtschaftspolitik an der Haltung der Danziger Bevölkerung etwas ändert. Die Preußen führten Zölle ein, was eine Wiederherstellung der alten Handelsverbindungen zwischen der Stadt und dem damaligen Kongresspolen an der Weichsel entlang unmöglich machte. Auch weitere von Berlin ausgehende Handlungen, besonders die Favorisierung von Königsberg und Stettin zuungunsten von Danzig, konnten den Unmut der Danziger nur noch verstärken (vgl. WŁODARCZYK 1998b: 30).

Ernst Förstemann (1822-1906) erinnert sich an die Anfänge jener zweiten preußischen Regierungszeit wie folgt:

„In den zwanziger Jahren war noch keineswegs die lebendige Ueberlieferung an die 1793 aufgehörte freireichsstädtische Herrlichkeit erloschen, auch die Bevölkerung durchaus nicht ganz in das preußische Wesen aufgegangen. [...] Auch lag in den damaligen Danzigern noch vielfach die Anschauung, daß die erste, höchststehende Person in der Stadt der Oberbürgermeister v. Weickhmann sei, die Generale aber [...], der Regierungspräsident u. s. w. tiefer an Rang ständen.“ (FöRSTEMAnN 1900: 8-9)5

Die Danziger blickten mit Stolz auf die glanzvolle Vergangenheit ihrer Heimatstadt zurück. Sie mokierten sich über die preußischen Beamten, und noch in den 1840er Jahren war die Abneigung gegen die Preußen unter den Bewohnern spürbar (vgl. LoEw 2003: 81). Franz August Brandstädter, Professor des Akademischen Gymnasiums, fasste diese Einstellung in folgenden Worten zusammen: „Es musste eine ziemliche Zeit vergehen, ehe Misstrauen

4 Publikandum, Danzig den 3ten Februar 1814. Vgl. WŁodarczy (1998b: 40).

5 Die Stadtverwaltung blieb nach wie vor in den Händen alteingesessener Danziger Patrizier-Familien, denen Joachim Heinrich Weickhmann, Oberbürgermeister in den Jahren 1817-1850, sowie Karl August Groddeck, dessen Nachfolger in den Jahren 1851-1862, entstammten. 
und Widerwille gegen alles Preußische, namentlich die Königlichen Beamten, in der Masse der Bevölkerung erstarb” (BRANDSTÄDTER 1879: 325).

Die Danziger hatten noch gut in Erinnerung, dass ihre Stadt zur Zeit der polnischen Adelsrepublik ihren größten Reichtum erlangt hatte, und dass ihre Bürger damals der europäischen Kulturelite angehörten. In der neuen Situation, in der sich die Einwohner nun zurechtfinden mussten, legten sie immer wieder ihre Verbundenheit mit der Tradition an den Tag (vgl. CzyŻaK 1998: 185). ${ }^{6} \mathrm{Zu}$ einem späteren Zeitpunkt, als schon die nächste Generation der Danziger herangewachsen war, stellte der polnische Romancier Józef Ignacy Kraszewski bei seinem Besuch an der Mottlau fest, man solle „die Danziger zu schätzen wissen, da sie alten Kunstwerken und Erinnerungsstücken aus vergangenen Zeiten immer mit viel Achtung begegneten und diese mit lobenswerter Fürsorge umgaben" (vgl. KRASZEWSKI 1867: 251).

\section{Der „V̈̈lkerfrühling“ (die Revolutionen 1848/49)}

Noch in den 30er Jahren des 19. Jahrhunderts identifizierte sich die Mehrheit der Danziger nicht mit Preußen. Der allmähliche Integrationsprozess schritt jedoch in den nachfolgenden Generationen voran, angetrieben bzw. begünstigt durch mehrere Faktoren: die Schulpflicht, den Wehrdienst, die Zuwanderung aus umliegenden Gebieten und die dauerhafte Präsenz von preußischen Beamten und Offizieren in der Stadt (vgl. CieślaK / Biernat 1994: 370).

Die Gesinnung der Danziger kam infolge der Ereignisse des „Völkerfrühlings“ 1848 besonders deutlich zum Ausdruck. Der Aufstand in Großpolen erfüllte die Danziger Bevölkerung mit Sorge über die Zukunft ihrer Heimatstadt und erweckte ihren Widerspruch gegen die polnischen Ansprüche auf das ehemalige Königliche Preußen. Am 3. April 1848 verfassten das Danziger Magistrat und die Stadtverordneten eine Adresse an den Deutschen Bund, in der sie die Einverleibung der vereinigten Provinz Preußen in den Bund forderten: „Deutsch sind wir und wollen es bleiben!“ (zit. nach LoEw 2011: 160). Oberbürgermeister war damals Joachim Heinrich von Weickhmann, der in den Augen der Stadtbewohner den Kampf um Danzigs Identität geradezu verkörperte (vgl. WŁODARCZYK 1998a: 167).

\section{Dritte Generation: 1849-1871}

Allmählich verkam das einst blühende internationale Handelszentrum zu einer Provinzstadt, in der Beamte und Offiziere mit ihren Familien 20\% der Einwohner ausmachten. Gleichzeitig war die Mentalität der Danziger im Wandel begriffen. Wie sich vermuten lässt, war die lokale Identität noch in den 1850er Jahren in zwei Hälften gespalten: Einerseits gab es die Alt-Danziger, andererseits - die zugewanderten Anhänger der „preußischen Option“ (vgl. LoEW 2003: 103). Wie sich bald herausstellte, gehörte die Zukunft der letztgenannten Gruppe.

6 Johann Carl Schultz, ein prominenter Maler und Graphiker sowie Leiter der Danziger Kunstschule, bedauerte in einem öffentlichen Vortrag, dass die Porträts der polnischen Könige, die sich im Weißen Saal des Rechtstädtischen Rathauses befanden, unlängst von dort entfernt worden seien (vgl. SCHULTZ 1841: 21). 
Im Laufe der Zeit mussten sich die traditionsliebenden Alt-Danziger damit begnügen, der vergangenen Epoche der souveränen Stadtrepublik melancholisch nachzuhängen. Diese Stimmung hielt der Reiseschriftsteller Ludwig (Louis) Passarge (1825-1912) fest:

„Wie in Venedig lebt auch hier noch das Bewußtsein der einstigen Größe nicht bloß unter den früheren Herrschern, sondern auch im Volke. Und obwohl Danzig sich unter der Herrschaft des preußischen Königshauses wohl fühlt, so ist es doch nichts weiter als das Gefühl der Sicherheit nach dem Beraubtsein." (PASSARge 1857: 58)

Das Gefühl, dass die alte Danziger Seele im Verschwinden begriffen war, muss für den Teil der Bevölkerung mit einem ausgeprägten Identitätsbewusstsein spürbar gewesen sein. Davon zeugt beispielsweise der folgende dramatische Aufruf: „Danziger, macht die Tore zu, damit das alte Danzig nicht ganz hinausgehe".

Die Einigung Deutschlands 1871 brachte eine wesentliche Veränderung für die Situation Danzigs mit sich. Mit dem Aufschwung des Seehandels nach der Aufhebung des Sundzolls 1857 erhielt die wirtschaftliche Entwicklung der Stadt einen deutlichen Schub. Dennoch meinte der ungarische Linguist Pál Hunfalvy (Paul Hunsdorfer), der das Erscheinungsbild Danzigs 1869 bewunderte, dass die Stadt an der Mottlau „sich unserm Jahrhundert noch nicht anschmiegen wollte oder nicht könnte; von welcher wir aber gleich herzlich gerne bekennen, dass sie daran wohl thut, so lange als möglich das zu bleiben was sie ist“"(Hunfalvy 1874: 1).

In der Tat sollte die materielle Substanz der Stadt noch lange bestehen bleiben, ihr innerer Geist hatte sich aber unwiederbringlich verflüchtigt. Zum Symbol der neuen Epoche wurden die Feierlichkeiten anlässlich des 100. Jubiläums der Eingliederung von Westpreußen in die Preußische Monarchie, die der Oberbürgermeister Leopold Winter 1872 in Marienburg veranstaltete. ${ }^{8}$ Die Alt-Danziger hätten dieses Ereignis nicht bejubelt, ihre Nachfolger aber machten nur noch eine Minderheit aus. Zudem wanderten die Nachkommen von alteingesessenen Patrizierfamilien nach und nach aus. ${ }^{9}$ Bald darauf stellte die Berliner Regierung, von der Loyalität der Danziger nun überzeugt, 1878 die ehemalige Provinz Westpreußen wieder her.

\section{Epilog}

Es ist schwer zu sagen, mit welcher der nachfolgenden Generationen die letzten echten Danziger verschwunden waren - die noch zu Beginn des 20. Jahrhunderts an der Mottlau lebten. ${ }^{10}$

7 Anonymer Druck Es gibt keine Danziger mehr! von 1867, zit. nach DomansKy (1923: 47).

8 Leopold von Winter, Oberbürgermeister von Danzig in den Jahren 1862-1890, hat sich um die Modernisierung der Stadt sehr verdient gemacht. Dennoch war er, der aus dem pommerschen Schwetz an der Weichsel stammte, der erste Nicht-Danziger, der dieses Amt bekleidete (vgl. GLIŃsKI 1994: 102-103).

91880 wanderte Max Carl Georg von Gralath (1851-1901), Angehöriger eines im 18. Jahrhundert sehr verdienten Danziger Geschlechts, nach Italien aus. Er nahm zahlreiche Familienerbstücke mit, die erst 2011 nach dem Tod des Raffaele Donati von Gralath in die Stadt zurückkehrten (vgl. OgONowska 2017: 276-277).

10 Nach hundert Jahren, die seit Danzigs Wiedereingliederung in Preußen 1815 vergangen waren, begegnete der polnische Historiker Jan Antoni Wilder einer Familie, die bei jeder Gelegenheit betonte, weder Preußen noch Deutsche, sondern Danziger zu sein (vgl. Wilder 1954: 501, Fußnote 1). 
An ihrer Stimme hatte es jedenfalls gefehlt, als die Gründung der historisch zweiten Freien Stadt Danzig 1920 auf allgemeinen Widerstand der preußisch gesinnten Danziger stieß.

Die Stadtverordnetenversammlung verkündete in ihrem Beschluss vom 13. Mai 1919 einstimmig Folgendes:

„Wir lehnen die Bildung eines Freistaates Danzig ab, weil wir darin nur die Vorstufe der Einverleibung Danzigs in Polen erblicken. Wie aber Danzig seit hunderten von Jahren deutsch fühlt und denkt, so will es auch jetzt dem deutschen Vaterland, mit dem es die guten Zeiten geteilt hat, die Treue wahren und es in seiner schwersten Not nicht verlassen." ${ }^{\text {"11 }}$

Die Danziger Deutschen, deren Wunsch nach der Rückkehr zum „deutschen Mutterland“ zwanzig Jahre später in Erfüllung ging, sollten die Vorteile der Wiederherstellung der politischen Verhältnisse, die ein Jahrhundert zuvor geherrscht hatten, erst am Vortag des Untergangs des Dritten Reichs 1945 erkennen. ${ }^{12}$ Doch für diese Einsicht war es zu spät. Die Geschichte des alten Danzigs kam zu einem Ende, weil die Stadt ihre Seele verloren hatte. ${ }^{13}$

Aus dem Polnischen von Katarzyna Lukas

\section{Bibliographie}

Askenazy, Szymon (1923): Gdańsk a Polska [Gdańsk / Danzig und Polen]. Warszawa: Gebethner i Wolff.

Biernat, Czesław (1993): Sytuacja polityczna i społeczna [Gdańska] [Zur politischen und gesellschaftlichen Lage Danzigs]. In: CieśLAK, Edmund (Hg.): Historia Gdańska [Geschichte von Danzig / Gdańsk] Bd. 3, T. 2: 1793-1815. Gdańsk: Wydawnictwo Morskie, 72-87.

Boennert, Günther (1922): Hindenburg in Marienburg. In: Danziger Allgemeine Zeitung $1922 \mathrm{Nr}$. 127 (1. Juni 1922), 1-2.

BoniSŁaWSKI, Wojciech / BALIŃski, Aleksander (Hg.) (2014): Gdańsk i okolice 1793-1914: miasto, ludzie, wydarzenia w rysunku i grafice [Danzig und die Umgebung 1793-1914: Die Stadt - Menschen - Ereignisse in Zeichnungen und Grafiken]. Gdańsk: Muzeum Narodowe.

BRANDSTÄDTER, Franz August (1879): Land und Leute des Landkreises Danzig: eine topographisch-historisch-statistische Schilderung [...]. Danzig: Verlag von Theodor Bertling.

CZYŻAK, Bogdan (1998): Starogdański nurt kultury [Der Alt-Danziger Kulturstil]. In: CIEŚLAK, Edmund (Hg.): Historia Gdańska [Geschichte von Danzig / Gdańsk] Bd. 4, T. 1: 1815-1920. Sopot: Wydawnictwo Lex, 185-239.

11 Protokoll der Stadtverordneten-Versammlung vom 19.05.1919, [75-76], zit. nach Loew (2011: 187). Nach der Gründung der Freien Stadt Danzig erklärte der stellvertretende Senatspräsident Ernst Ziehm dem Generalfeldmarschall Paul von Hindenburg in Marienburg: „Wir warten auf den Augenblick, wo wir wieder zu unserem Vaterlande gehören werden." Vgl. BOEHNERT 1922: 2, Spalte 2.

12 In den ersten Monaten des Jahres 1945 zählten etliche Bewohner immer noch darauf, nach Kriegsende wieder in einer Freien Stadt leben zu dürfen (vgl. Loew / ZeKeRT / RUSAK (Hg.) 1997: 152, 153).

13 Der Genius Loci der Danziger Unabhängigkeit und Selbstbestimmung offenbarte sich erneut während der Arbeiterstreiks in der Danziger Werft im Dezember 1970 und August 1980; aus den Streiks ging die freie Gewerkschaft „Solidarność” hervor. Ist dies tatsächlich eine ganz andere Geschichte? 
Declaration Seiner Majestät des Königs von Preussen, die Besetzung der Stadt und des Gebiets von Danzig betreffend. In: Girtanner, Christoph (Hg.): Politische Annalen Bd. 2. Berlin 1793: Johann Friedrich Unger, 102-103.

Domansky, Walther (1923): Alte Danziger. Lebensbeschreibungen. Danzig: Danziger Verlags-Gesellschaft.

Förstemann, Ernst Wilhelm (1900): Aus dem alten Danzig (1820-1840). Danzig: Verlag von L. Sanniers Buch- u. Kunsthandlung.

GŁęBOwicz, Bogdan (1961): Związek Wolnych Prusaków w Gdańsku (tzw. Spisek Gotfryda Beniamina Bartholdiego) [Das Komplott von Gottfried Benjamin Bartholdy]. In: Zapiski Historyczne (Toruń) 1961, Bd. XXVI, H. 1, 18-30.

GlińsKi, Mirosław (1994): Ludzie dziewiętnastowiecznego Gdańska [Die Menschen von Danzig im 19. Jh.]. Gdańsk: Muzeum Historii Miasta Gdańska.

Hunfalvy, Pál (1874): Reise in den Ostseeprovinzen Russlands. Leipzig: Duncker \& Humblot.

KRASZEW SKI, Józef Ignacy (1867): Listy (Z podróży): List II [Reisebriefe: Brief II]. In: Ktosy (Warszawa) Bd. V, 1867, Nr. 124 [2 (14) November 1867], 251.

LoEw, Peter Oliver (2011): Danzig. Biographie einer Stadt. München: Beck.

Loew, Peter Oliver (2003): Danzig und seine Vergangenheit 1793-1997. Die Geschichtskultur einer Stadt zwischen Deutschland und Polen. Osnabrück: Fibre.

Loew, Peter Oliver / Zekert, Reiner / Rusak, Elżbieta (Hg.) (1997): Danzig 1945. Erinnerungen nach 50 Jahren - Gdańsk 1945. Wspomnienia 50 lat później. Gdańsk: Marpress.

OgonowsKa, Ewa (2017): Biblia rodziny Gralathów [Die Bibel der Familie Gralath]. In: BALIŃsKI, Aleksander / FrącKowska, Anna / OTto, Maria (Hg.): Kronika Biblioteki Gdańskiej 1596-2016 [Chronik der Danziger Bibliothek 1596-2016]. Gdańsk: PAN Biblioteka Gdańska, 276-277.

Passarge, Louis (1857): Aus dem Weichseldelta. Reiseskizzen. Berlin: Verlag der Königlichen Geheimen Ober-Hofbuchdruckerei (R. Decker).

Publikandum, Danzig den 3ten Februar 1814. PAN Biblioteka Gdańska, Sign. Od 5740, 2ªdl. 208.

Rozenkranz, Edwin (1980): Napoleońskie Wolne Miasto Gdańsk. Ustrój, prawo, administracja [Die napoleonische Freie Stadt Danzig. Struktur, Rechtssystem, Verwaltung]. Wrocław, Gdańsk: Ossolineum.

Schopenhauer, Johanna (1884): Jugendleben und Wanderbilder. Danzig: Verlag von Theodor Bertling.

Schultz, J[ohann] C[arl] (1841): Ueber alterthümliche Gegenstände der bildenden Kunst in Danzig.... Danzig: Gerhardsche Offizin.

Wilder, Jan Antoni (1954): Stosunek Gdańszczan do Prus w przededniu Kongresu Wiedeńskiego [Zur Einstellung der Danziger zu Preußen am Vorabend des Wiener Kongresses]. In: Przegląd Zachodni (Poznań) 1954, X, Bd. 3, Nr. 10, 501-518.

WŁodARCZyK, Edward (1998a): Kształtowanie się nowoczesnego społeczeństwa miejskiego [Zur Entwicklung der modernen urbanen Gesellschaft]. In: Cieślak, Edmund (Hg.): Historia Gdańska [Geschichte von Danzig / Gdańsk] Bd. 4, T. 1: 1815-1920. Sopot: Wydawnictwo Lex, 146-169.

WŁODARCZYK, Edward (1998b): Miejsce Gdańska w państwie pruskim [Zur Stellung Danzigs im preußischen Staat]. In: Cieślak, Edmund (Hg.): Historia Gdańska [Geschichte von Danzig / Gdańsk] Bd. 4, T. 1: 1815-1920. Sopot: Wydawnictwo Lex, 23-49.

ZAJEWSKI, Władysław (1993a): Stagnacja gospodarcza i eksploatacja finansowa Wolnego Miasta Gdańska [Die wirtschaftliche Stagnierung und finanzielle Ausbeutung der Freien Stadt Danzig]. 
In: CieślaK, Edmund (Hg.): Historia Gdańska [Geschichte von Danzig / Gdańsk] Bd. 3, T. 2: 1793-1815, Gdańsk: Wydawnictwo Morskie, 150-167.

ZAJEWski, Władysław (1993b): Zabiegi Gdańska o rozpatrzenie losów miasta na Kongresie Wiedeńskim 1814-1815 r. [Die Bemühungen Danzigs darum, die Zukunft der Stadt auf dem Wiener Kongress 1814-1815 zu diskutieren]. In: CIEŚLAK, Edmund (Hg.): Historia Gdańska [Geschichte von Danzig / Gdańsk] Bd. 3, T. 2: 1793-1815, Gdańsk: Wydawnictwo Morskie, 188-196. 